“ (C) 2019 IEEE. Personal use of this material is permitted. Permission from IEEE must be obtained for all other uses, in any current or future media, including

reprinting/republishing this material for advertising or promotional purposes, creating new collective works, for resale or redistribution to servers or lists, or reuse of any copyrighted component of this work in other works." 


\title{
Suppression of Cross-Band Scattering in Multiband Antenna Arrays
}

\author{
Hai-Han Sun, Student Member, IEEE, Can Ding, Member, IEEE, He Zhu, Member, IEEE, \\ Bevan Jones, Life Member, IEEE, and Y. Jay Guo, Fellow, IEEE
}

\begin{abstract}
This paper presents a novel method of suppressing cross-band scattering in dual-band dual-polarized antenna arrays. The method involves introducing chokes into low-band (LB) elements to suppress high-band (HB) scattering currents. Experimental results show that by inserting LB-pass HB-stop chokes into LB radiators, suppression of induced $\mathrm{HB}$ currents on the LB elements is achieved. This greatly reduces the pattern distortion of the HB array caused by the presence of LB elements. The array considered is configured as two columns of $\mathrm{HB}$ antennas operating from $1.71 \mathrm{GHz}$ to $2.28 \mathrm{GHz}$ interleaved with a single column of $\mathrm{LB}$ antennas operating from $0.82 \mathrm{GHz}$ to $1.0 \mathrm{GHz}$. The realized array with choked $\mathrm{LB}$ element has stable and symmetrical radiation in both high and low bands.
\end{abstract}

Index Terms - Dual band antenna arrays, interleaved arrays, pattern distortion, scattering suppression.

\section{INTRODUCTION}

$\mathrm{O}$ WING TO the ever-increasing demand on wireless communications and sensing, there has been a growing interest in multiband antennas to simultaneously support different services. The arrays for different bands usually share a common ground plane and radome, and the elements of different bands are often interleaved to save space. However, there is a penalty for the close proximity of the elements in the form of distortion of the pattern due to scattering of the signals of one band by the antenna elements operating in another band [1]. Since low-band (LB) antenna elements are typically larger than high-band (HB) ones, suppression of the scattering of $\mathrm{HB}$ signals from LB elements can significantly improve the system performance.

In this paper, we consider a multiband $3 \mathrm{G}$ and $4 \mathrm{G}$ base station antenna (BSA) array as a typical example. Such antennas need at least a LB antenna array operating from 0.69 $\mathrm{GHz}$ to $0.96 \mathrm{GHz}$, and a $\mathrm{HB}$ antenna array operating from 1.71 $\mathrm{GHz}$ to $2.69 \mathrm{GHz}$. The $\mathrm{LB}$ and $\mathrm{HB}$ antenna arrays share the same area to minimize the space rental cost. As the HB frequencies are almost one octave above the LB frequencies, the $\mathrm{HB}$ and LB elements are usually interleaved in a uniform way where the spacing of $\mathrm{HB}$ elements is half that of the LB elements. Common arrangements of HB and LB dual-polarized elements in arrays are shown in Fig. 1. In Fig. 1(a), every second $\mathrm{HB}$ element is embedded in a physically larger LB

Manuscript submitted xxxx xxxx, 2018. element, which is referred as "embedded scheme". Many dualband dual-polarized arrays with this scheme are described in [2]-[9]. Different shapes of metal walls are added to control the radiation patterns and improve isolation between $\mathrm{LB}$ and $\mathrm{HB}$ elements. An alternative configuration is the "interleaved scheme" shown in Fig. 1(b), where one LB column is located midway between the two HB columns. This configuration is common in practice as it provides multiple-input and multipleoutput (MIMO) capability in high band and low band in a minimum width package [10]. A problem with this configuration is that because of the proximity of the HB and LB elements, and due to the electrically large dimension of the LB element at high band, HB currents are induced on the LB element which radiate unwanted signal at high band. This scattered signal causes major distortion of the $\mathrm{HB}$ radiation pattern.

The reverse effect of distortion of the LB pattern due to the presence of the HB elements can occur if the HB elements have a resonance within or adjacent to the low band. It normally occurs in a relatively narrow band around the LB resonance but can have a serious effect on the LB azimuth pattern. However, this generally can be corrected by moving the resonance out of the low band of interest. This issue is not considered in this paper.

One method of minimizing scattering where closely spaced radiators operate at different frequencies makes use of mantle cloaking techniques, as described in [1], [11]-[15]. These mantle cloaks are designed to produce an "anti-phase" scattering currents to cancel the scattering from radiator alone [11]. They can largely suppress cross-band scattering while preserving performance of the cloaked radiator. In [1] and [13], cloaks are designed for use in simple BSA cases.

In this paper, a different technique is used to minimize scattering in multi-band arrays. This method relies on modifying the LB radiator itself by introducing chokes which effectively divide the conductors of the LB elements into short sections. These sections are significantly shorter than resonant length at the $\mathrm{HB}$ and so have much reduced currents induced in them. Cross-dipoles described in [16], [17] are used as the LB elements to demonstrate the effectiveness of the method. Chokes that present open circuits at high band and short circuits at low band are used to bridge a number of cuts in the LB dipole

The authors are with the Global Big Data Technologies Center, University of Technology Sydney, Sydney, NSW 2007, Australia (email: Haihan.Sun199403@gmail.com) 
arms, forming a LB element with reduced $\mathrm{HB}$ currents but with LB performance largely maintained. Although the inserted chokes change the impedance of the LB element, making matching more difficult, satisfactory impedance matching is obtained using baluns and impedance transformers designed following the guidelines given in our previous work [18], [19]. In the reported research, a section of the dual-band dualpolarized array with chokes was constructed and tested. Simulated and measured results demonstrate that addition of the chokes greatly reduces scattering while retaining stable radiation properties across the two bands.

The array in this paper is designed to operate at low band ranging from $820 \mathrm{MHz}$ to $1000 \mathrm{MHz}$ and the high band from $1710 \mathrm{MHz}$ to $2280 \mathrm{MHz}$ - which cover the operating frequencies of most mobile communication systems, including CDMA, GSM, PCS, and UMTS. Future work will concentrate on broadening the bandwidth to cover the full $4 \mathrm{G}$ bands.

The paper is arranged as follows. Section II demonstrates the scattering problem in the dual-band dual-polarized BSA array section by examining the HB pattern distortion caused by a simple interleaved LB element. Section III discusses the operating principle and the design procedure of the choked LB element. The HB pattern with choked LB element is presented to demonstrate that choking LB arms can suppress the HB scattering effectively. The matching capability and the radiation performance of the choked LB element are also presented to verify that choking the LB arms does not degrade its original performance. Section IV presents the simulated and measured results of the dual-band dual-polarized antenna array with choked LB element.

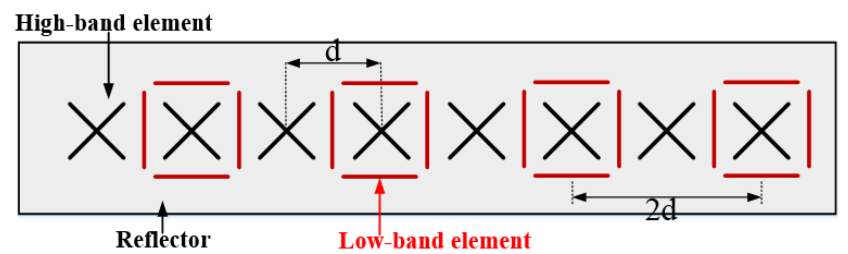

(a)

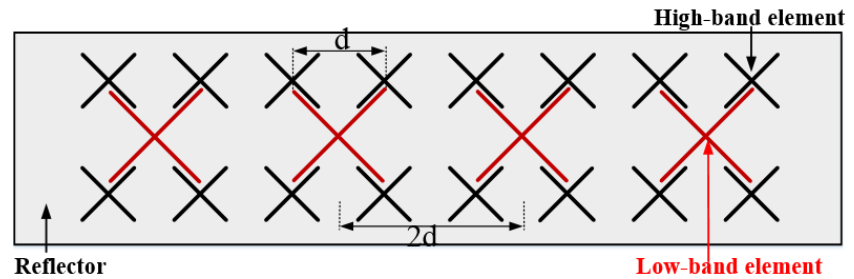

(b)

Fig. 1. Dual-band dual-polarized base station antenna array configurations with (a) embedded scheme, and (b) interleaved scheme.

\section{Problem Statement}

To begin with, a section of the interleaved dual-band BSA array shown in Fig. 2 using unaltered LB elements is simulated to demonstrate the scattering problem. The complete BSA array consists of array sections repeated in the y-direction. In this array, a strip-shaped cross-dipole is used as the unaltered LB element, and square-shaped cross-dipoles are used as the HB elements. Baluns are used to provide balanced feeding and impedance matching for these elements. The LB element is located midway between the four HB elements in two columns. The two HB columns form two HB sub-arrays, and they are fed from independent wideband phase-shifters which for modeling purposes are represented as power dividers, as shown in Fig. 2. (b). HB elements with the same polarizations in one column are excited simultaneously. The LB element is fed separately at the inputs to the two baluns. The parameters for the array arrangement are marked in Fig. 2. Those parameters were chosen for good MIMO performance while keeping the array compact. The S-parameters for the LB and HB elements when alone are shown in Fig. 3. The LB element operates from 0.82 $\mathrm{GHz}$ to $0.98 \mathrm{GHz}$, and the $\mathrm{HB}$ element operates from $1.70 \mathrm{GHz}$ to $2.30 \mathrm{GHz}$.

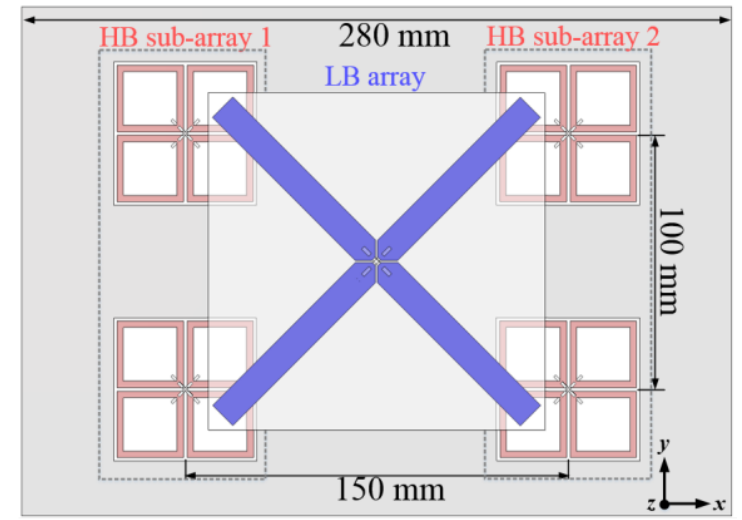

(a)

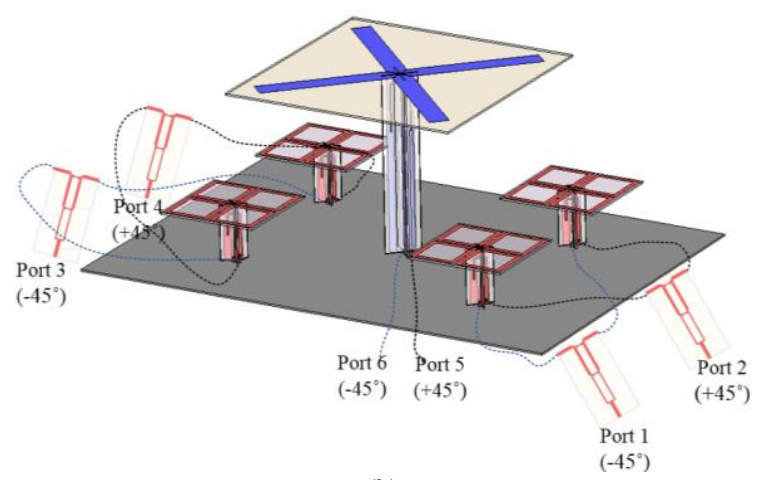

(b)

Fig. 2. Configuration of the interleaved BSA array section: (a) top view, and (b) perspective view.

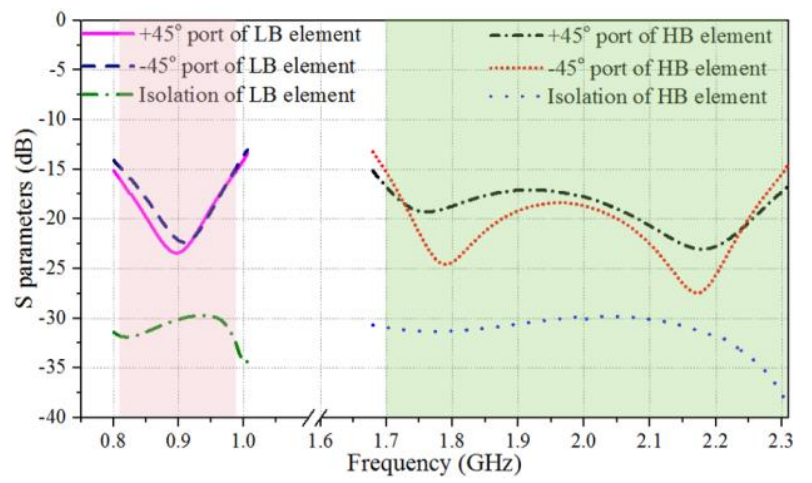

Fig. 3. Simulated S-parameters for the LB and HB antennas when working alone.

It is apparent from Fig. 2(a) that the LB element overlaps the 
HB elements. The close spacing between LB and HB elements causes scattering of HB signal by the LB element. The simulated current distribution in the array with unaltered LB element at $1.7 \mathrm{GHz}$ is shown in the Fig. 4. Most of the induced current on the LB arms has the same direction as the HB driven current. These currents re-radiate, resulting in a deteriorated $\mathrm{HB}$ radiation pattern. The radiation patterns in the horizontal plane (xoz-plane) with and without the LB element are shown in Fig. 5 at $1.7 \mathrm{GHz}, 1.8 \mathrm{GHz}$ and $1.9 \mathrm{GHz}$. Without the LB element, the $\mathrm{HB}$ array has a symmetrical pattern with main beam pointing at boresight. After the LB element is added, the HB radiation pattern deteriorates, i.e. the main beam splits or shifts away from boresight. In the worst case, the main lobe direction of the pattern is tilted to $19^{\circ}$, as shown in Fig. 5(a). The distorted radiation patterns obtained after adding the LB element cannot provide required coverage, and lead to signal loss in particular areas. This is generally unacceptable by cellular operators. It is therefore very desirable to find a method of suppressing the scattering and restoring the pattern in such multiband BSAs.

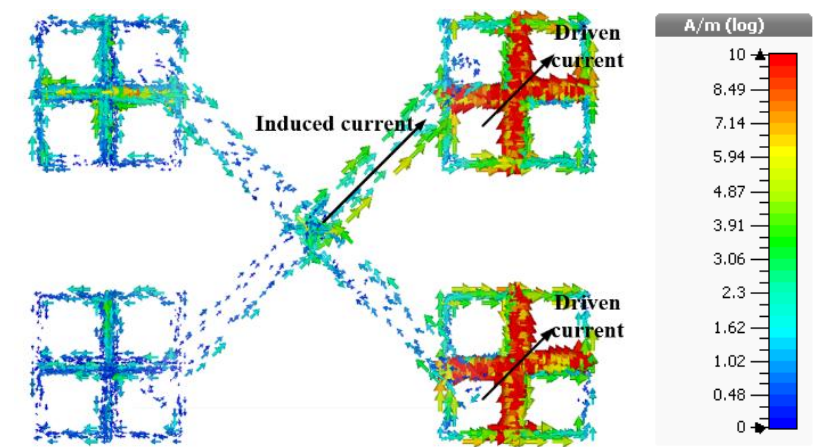

Fig. 4. Current distribution when HB array on the right column is excited.
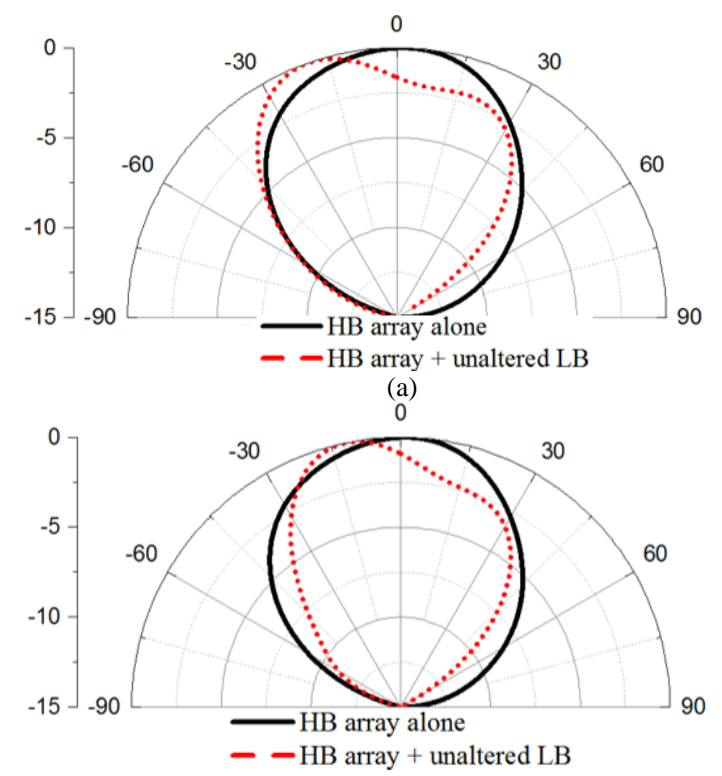

(b)

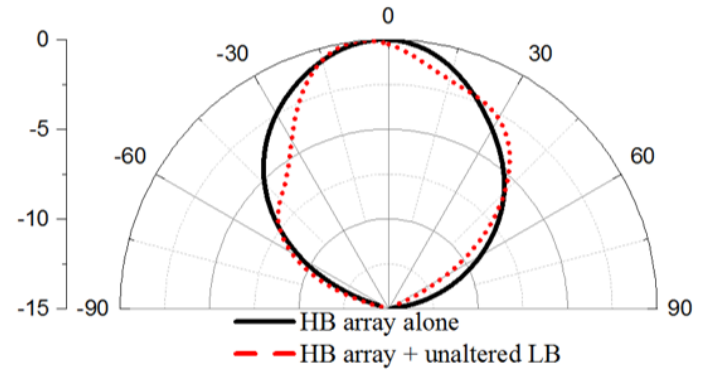

(c)

Fig. 5. Horizontal radiation patterns of the HB array i) without, and ii) with the unaltered LB element at (a) $1.7 \mathrm{GHz}$, (b) $1.8 \mathrm{GHz}$, and (c) $1.9 \mathrm{GHz}$.

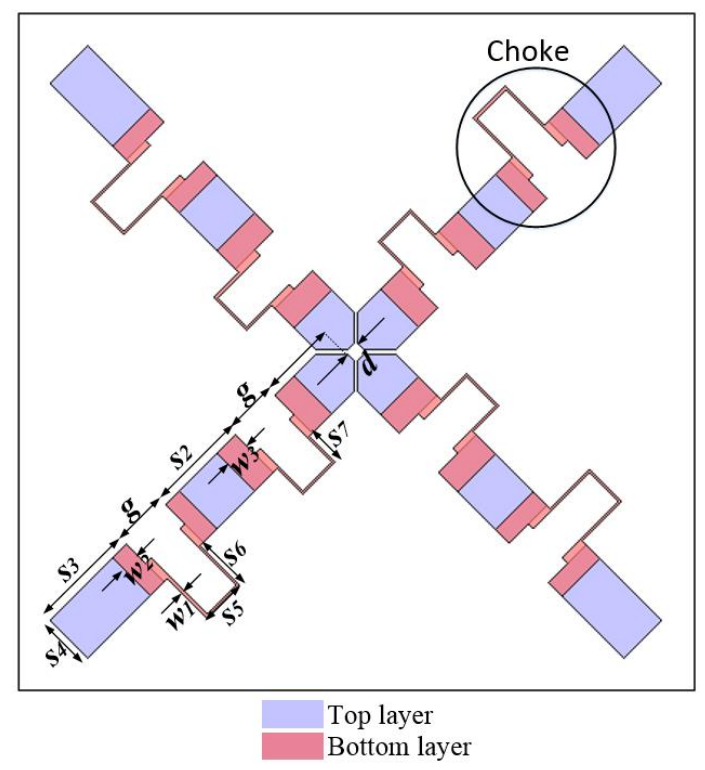

Fig. 6. Configuration of the choked radiator.

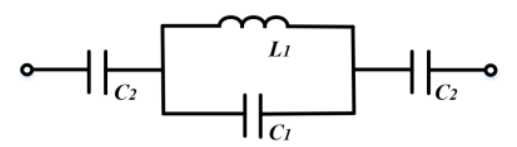

Fig. 7. Circuit model of the choke.

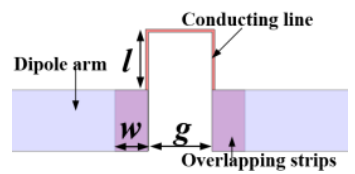

(a)

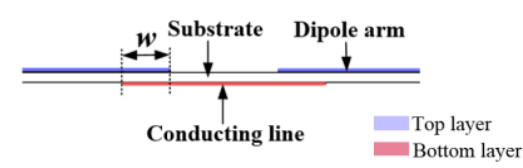

(b)
Fig. 8. (a) Top, and (b) side view of the realized choke.

\section{DESIGN OF CHOKED LOW-BAND ELEMENT}

To minimize the scattering shown above, one effective way is to suppress the induced HB currents on the LB arms. In this work, this is achieved by introducing chokes periodically along LB dipole arms to block the HB currents but affect LB currents as little as possible, as shown in Fig. 6. These chokes should present an open circuit at high band and a short circuit at low band. The design principle and the performance of the choked LB antenna are detailed in the following.

\section{A. Circuit Model of the Choke}

The circuit model of the choke is shown in Fig. 7. It consists of a parallel resonant at high band comprising $L_{l}$ and $C_{l}$, and a 
series resonance at low band with two additional capacitances $C_{2}$. These requirements give

$$
\begin{gathered}
j 2 \pi f_{h} C_{1}+\frac{1}{j 2 \pi f_{h} L_{1}}=0 \\
\frac{2}{j 2 \pi f_{l} C_{2}}+\frac{1}{j 2 \pi f_{l} C_{1}+\frac{1}{j 2 \pi f_{l} L_{1}}}=0
\end{gathered}
$$

where $f_{h}$ is the open-circuit frequency point at high band, and $f_{l}$ is the short-circuit frequency point at low band. The values of $f_{h}$ and $f_{l}$ are both known in our target application. A remaining variable can be chosen to determine values for all the components $L_{1}, C_{1}$ and $C_{2}$.

$$
Z_{c}=\sqrt{\frac{L_{1}}{C_{1}}}
$$

This equation sets the impedance level at which the choke operates and determines the bandwidths over which the open circuit and short circuit are effective. $L_{1}, C_{1}$, and $C_{2}$ can be found given $f_{h}, f_{l}$, and $Z_{c}$. By suitably choosing $L_{l}, C_{l}$, and $C_{2}$, we can attain desired open-circuit and short-circuit frequency points at high and low bands, respectively.

\section{B. Physical Realization of the Choke}

The choke circuit is realized by a conducting strip structure, as shown in Fig. 8(a) and (b). The thin conducting strip and the capacitance across the gap in the dipole arm provide $L_{l}$ and $C_{l}$. $C_{2}$ is realized by placing the inductive lines on the bottom layer of the substrate and adding strips that overlap with dipole arms on the top layer. The thin inductive line and gap capacitance control the open circuit at high band, and the overlapping strips (series capacitance) control the short circuit at low band. In this way, a geometry that approximately represents the circuit in Fig. 7 is designed. The choke is optimized following two criteria, i.e. to suppress the current at high band and to create a pass band at low band, which are discussed in subsection 1 and 2 , respectively. The number of the chokes to be inserted in the dipole arms is determined in the last step, which is shown in subsection 3 . The details of the optimization and analysis are as follows.

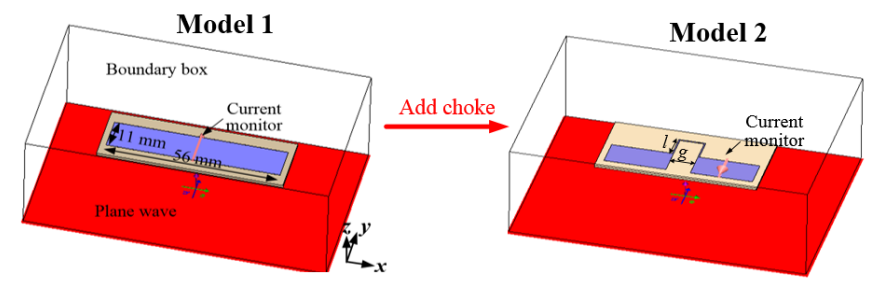

(a)

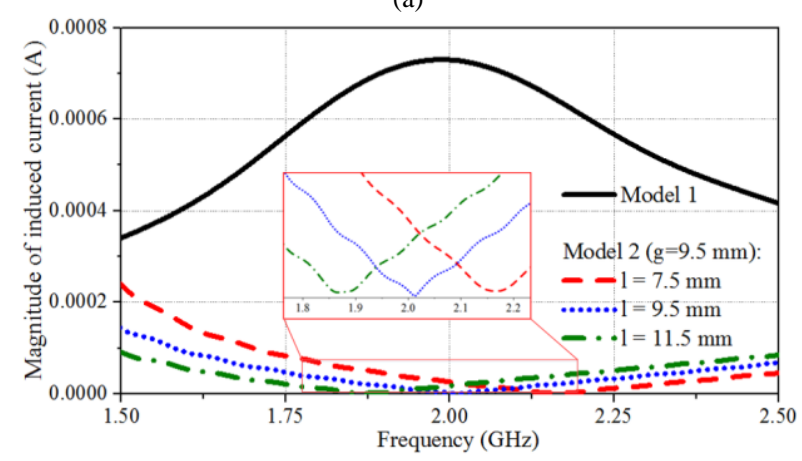

(b)

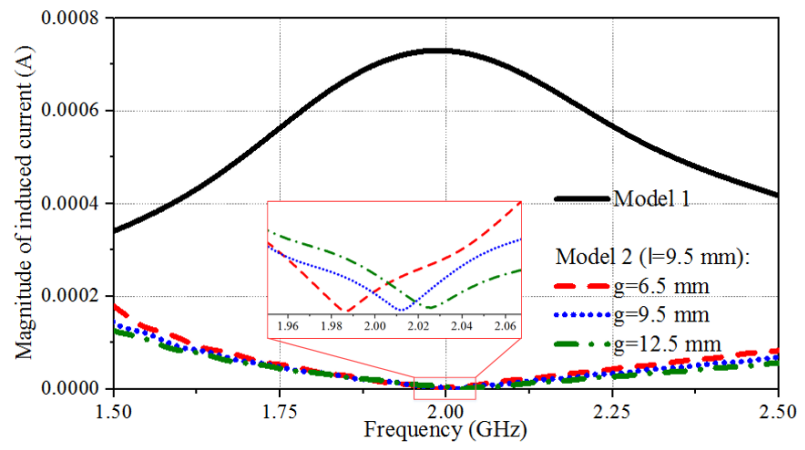

(c)

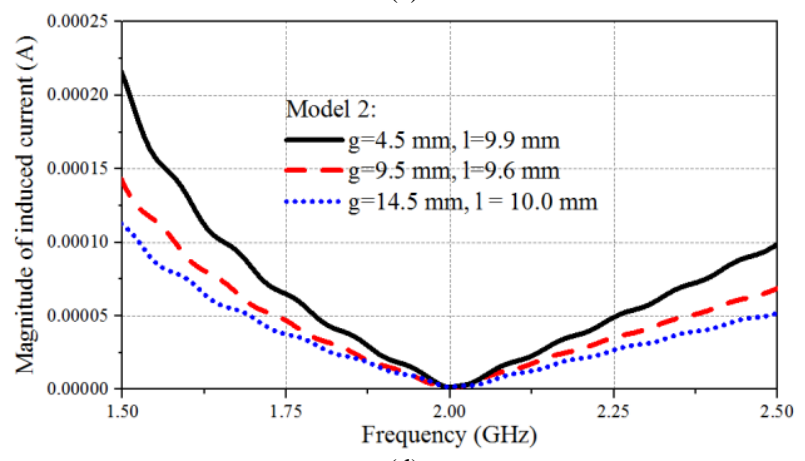

(d)

Fig. 9. (a) Model 1: a strip with a length of around $\lambda / 2$ at $2.0 \mathrm{GHz}$ (middle frequency at high band) and a width of $11 \mathrm{~mm}$; Model 2: the strip in Model 1 cut in the middle and bridged with an inductive line. (b) Comparison of induced HB current on the strip in Model 1, and on the strip in Model 2 with different value of $l$. (c) Comparison of induced HB current on the strip in Model 1, and on the strip in Model 2 with different value of $g$. (d) Comparison of induced HB currents on the strip in Model 2 with optimized $\{g, l\}$ values for open circuit point at $2.0 \mathrm{GHz}$.

\section{1) Optimization of the choke for HB suppression}

Firstly, the choke is optimized to suppress induced current at high band around 2.0 GHz. To assess the effect of the choke suppressing scattering, it is introduced into a strip representing a section of the dipole arm with a length around $\lambda / 2$ at $2.0 \mathrm{GHz}$, as shown in Fig. 9(a). ( $\lambda / 2$ is the resonant length including the effects of strip width and dielectric of the substrate.) Model 1 shows the strip without modification, and Model 2 shows a gap of width $g$ introduced at the center of the strip. An inductive line of length of $2 l+g$ is used to bridge the cut. This inductive line and the gap capacitance are used to realize $C_{l}$ and $L_{l}$ in the choke equivalent circuit. Guided by the circuit, adjusting $g$ and $l$ can effectively tune the open-circuit frequency point.

The models are illuminated by plane wave with E-field parallel to the length of the strip, and the maximum induced HB currents flowing on the strips are monitored. For Model 1, there a noticeable amount of HB current induced on the strip, and the induced current has its peak at around $2.0 \mathrm{GHz}$, as shown in Fig. 9(b). For Model 2, the maximum induced HB currents with different values of $g$ and $l$ are monitored. The results are plotted in Fig. 9(b) and (c). Both figures depict that Model 2 has much less amount of HB current induced on the strips than Model 1, which presents the scattering suppression capability of the choke.

The influences of parameters $g$ and $l$ are studied. As shown in Fig. 9(b), for a given $g$, increasing $l$ makes the minimum induced current point appear at a lower frequency, showing that 
the open-circuit point is moved to a lower frequency. This is because increasing $l$ increases $L_{l}$, making the $f_{h}$ lower. As shown in Fig. 9(c), for a given $l$, increasing $g$ reduces $C_{l}$ and moves the open-circuit point slightly to a higher frequency. To achieve open-circuit conditions at $2.0 \mathrm{GHz}$, different combinations of $g$ and $l$ can be chosen. The maximum magnitudes of current flowing on the strip with some suitable combinations are plotted in Fig. 9(d). It shows that combination with larger $g$ provides current suppression across a wider bandwidth. This is because increasing $g$ increases $Z c$ in (3) and widens the bandwidth of the suppression.

\section{2) Optimization of the choke for $L B$ pass}

After determining parameters $\{g, l\}$ having desirable scattering suppression at high band, the next step is to optimize the choke to minimize its influence at the operating low band around $0.89 \mathrm{GHz}$. In this step, a strip with a length of around $\lambda / 2$ at $0.89 \mathrm{GHz}$ is modeled with a choke at the center, as shown in Fig. 10(a). The inductive line is now placed on the bottom layer of the substrate and attached to two patches overlapping the cut segments on the top layer. The overlap width w determines $\mathrm{C} 2$ in the choke circuit. Adjusting it tunes the short-circuit frequency point.

The models are illuminated by plane wave at low band, and the maximum induced LB current is monitored. The results are shown in Fig. 10(b). For Model 3, the induced LB current has a peak value at $0.89 \mathrm{GHz}$. As for Model 4, similar amount of LB current is induced on the strip, but the frequency of the current maximum depends on the value of $w$. The frequency of the current maximum corresponds to the short-circuit point. Increasing w increases $C_{2}$ in the circuit, thus moves the shortcircuit point to a lower frequency. Therefore, for a given $\{g, l\}$, larger $\mathrm{w}$ moves the current maximum to a lower frequency in the simulation, which is shown in Fig. 10(b).

For different combinations of $\{g, l\}$ that can achieve opencircuit at $2.0 \mathrm{GHz}$, different values of $w$ are required to achieve short-circuit point at $0.89 \mathrm{GHz}$. The induced LB current on the strip of Model 4 with the some optimized $\{g, l, w\}$ combinations are shown in Fig. 10(c). Combination with larger $g$ have a narrower bandwidth at low band as they require a smaller value of $C_{2}$. Therefore, the combination of $\{g, l, w\}$ needs to be selected carefully to achieve the desired pass band at low band, and stop band at high band.

Model 3

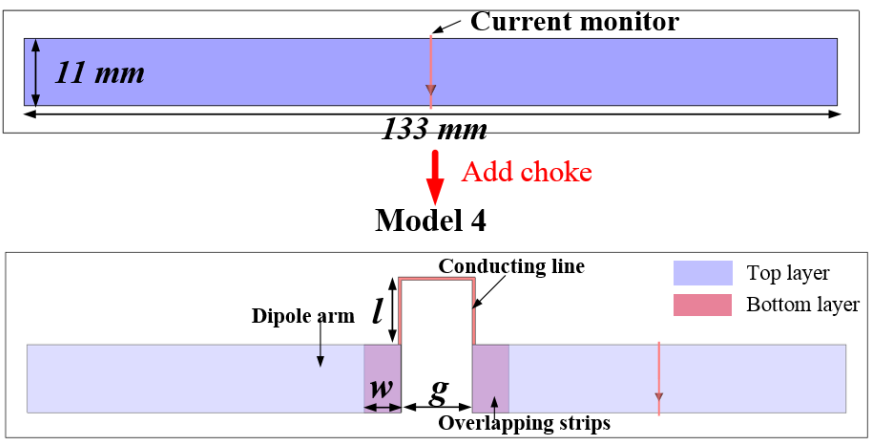

(a)

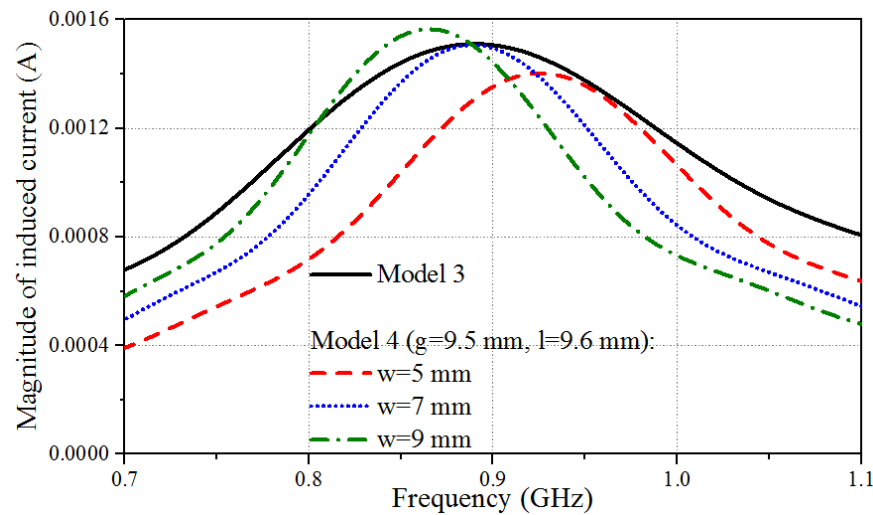

(b)

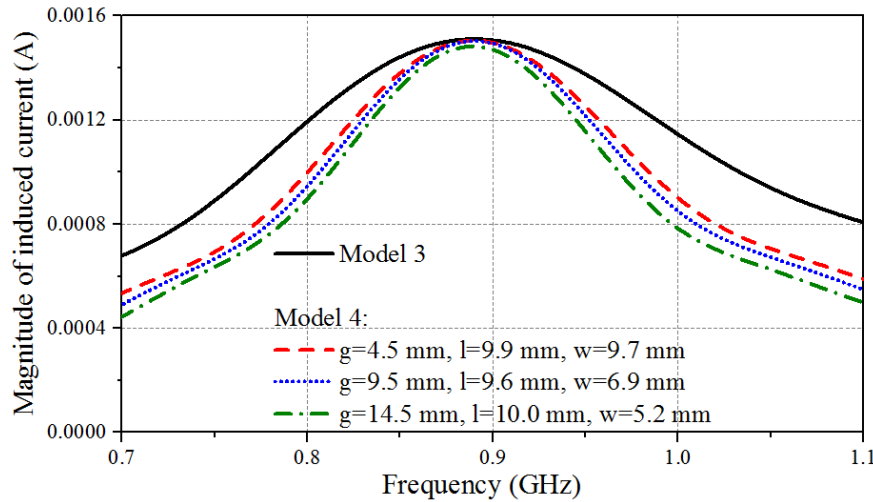

(c)

Fig. 10. (a) Model 3: a strip with a length of around $\lambda / 2$ at $0.89 \mathrm{GHz}$ (middle frequency at low band) and a width of $11 \mathrm{~mm}$; Model 4: the strip in Model 3 cut in the middle and bridged with a choke. (b) Comparison of induced LB current on the strip in Model 3, and on the strip in Model 4 with different values of $w$. (c) Induced LB current on the strip in Model 4 with optimized $\{g, l, w\}$ values for open circuit point at $2.0 \mathrm{GHz}$ and short circuit point at $0.89 \mathrm{GHz}$.

3) Determining the number of chokes required in the LB arms

The LB dipole arms are cut into several short segments with gaps between them, and the chokes described above are introduced into the gaps, as illustrated in Fig. 11. It is necessary to determine the number of chokes required in a dipole arm. The choke inevitably introduces some loss to the LB antenna, i.e. the magnitude of the current on a choke is slightly smaller than that on an unmodified strip as shown in Fig. 10(c). Therefore, unnecessary chokes should be avoided. On the other hand, using too few chokes reduces the level of current suppression at high band.

To determine the length of each segment $\left(l_{-} s\right)$ that assure suitably low HB currents on the segment, segments with different lengths are modelled with illumination by plane wave with E-field parallel to the length of the segment as shown in Fig. 12(a). The maximum induced HB current levels are monitored with results shown in Fig. 12(b). As expected, the shorter the segment is, the lower is the induced current. Segment lengths around $20 \mathrm{~mm}$ offer great reduction of the HB induced current. For the LB arms, two cuts with a gap width around $11 \mathrm{~mm}$ in each arm achieve that. Simulations confirm that the HB radiation pattern is almost unchanged with the cut LB arms. 


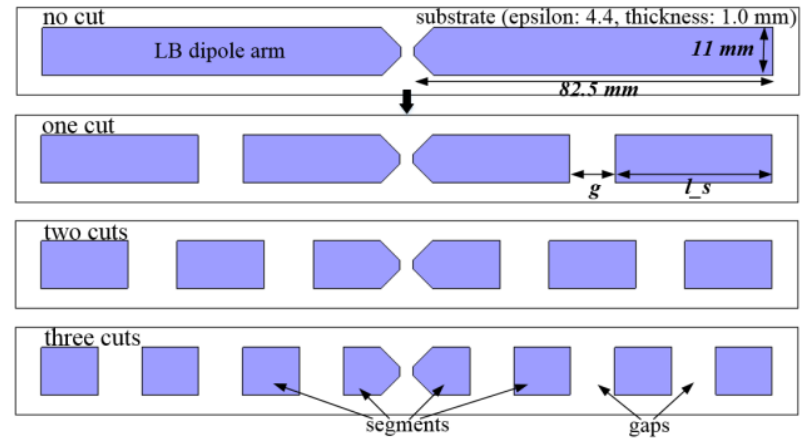

(a)

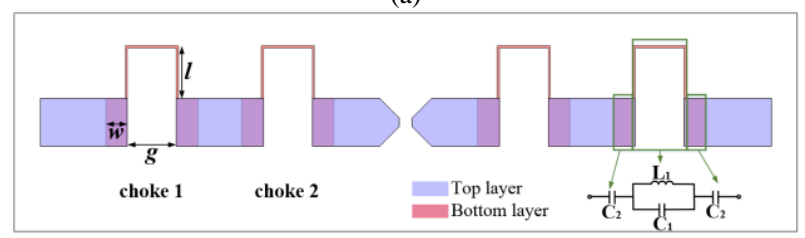

(b)

Fig. 11. (a) The LB dipole arms with different number of cuts. (b) Top view of the LB dipole arm with chokes inserted in.

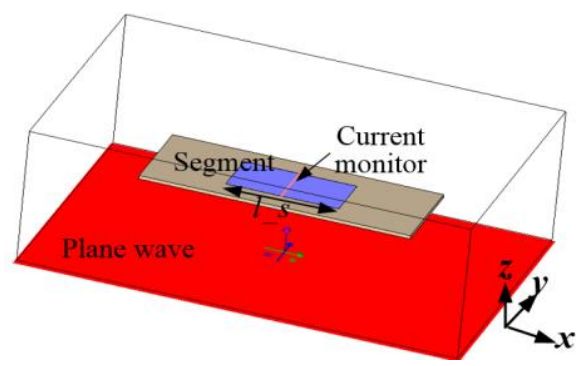

(a)

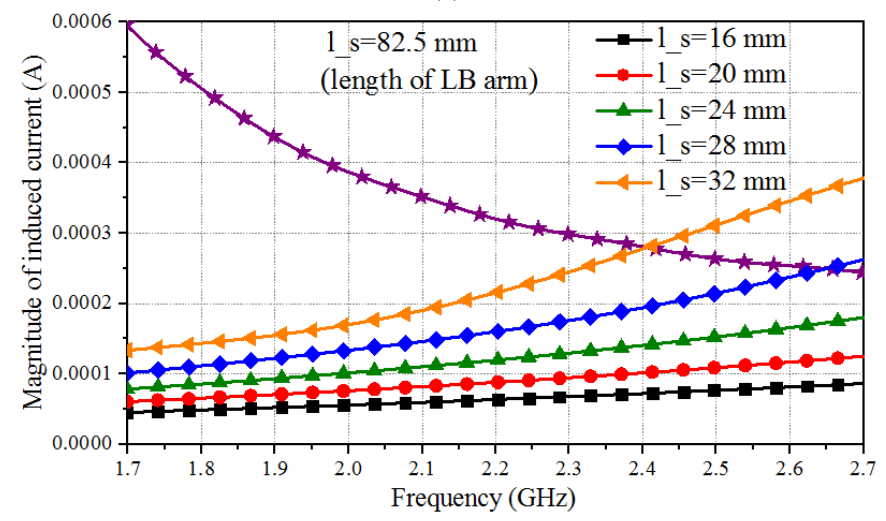

(b)

Fig. 12. (a) Model to determine effect of segment length $l \_s$ in free space illuminated by HB plane wave. (b) Maximum induced HB current on the segment with different $l_{-} s$.

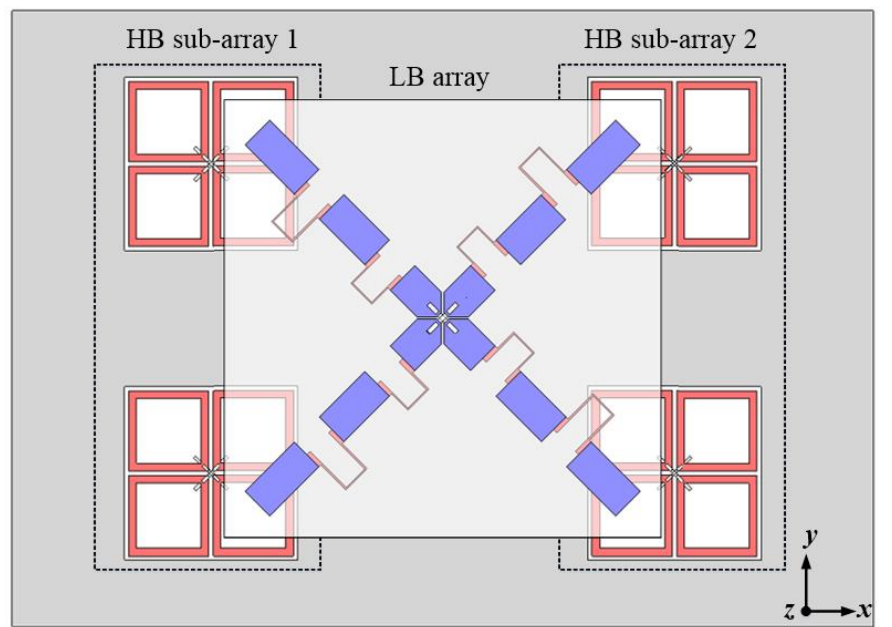

Fig. 13. Arrangement of the interleaved dual-band array with choked LB radiators.

\section{Scattering Suppression of the Choked LB Radiators}

The optimized choked LB radiators are shown in Fig. 6. To achieve HB choking performance across a wide band, two chokes tuned to slightly different frequencies were chosen and introduced into the two gaps in each LB arm. The choked LB arms are still arranged in the cross-dipole configuration to realize the required dual-polarization radiation. The optimized parameters are listed in Table I. The substrate for the radiator has a dielectric constant of 4.4 , a loss tangent of 0.0025 , and a thickness of $1.0 \mathrm{~mm}$. As the size of the choke in this design is relatively large, the orientation of the chokes is arranged in an anti-clockwise direction to minimize the coupling between inductors on different arms. If the chokes were realized with a smaller dimension, their orientation would be of no consequence.

The optimized choked LB radiators are introduced into the dual-band antenna array as shown in Fig. 13 to examine their effectiveness in suppressing the HB scattering in a realistic environment. Except for the modification of the LB arms, the arrangement of this array is unchanged from that of Fig. 2. HB elements in one column form one HB sub-array, and elements with the same polarizations in one sub-array are excited simultaneously using wideband power dividers. One column of choked LB elements is located midway between the two HB sub-arrays, forming an interleaved dual-band dual-polarized BSA array.

Fig. 14 shows plots of E-field in a horizontal section through the array across the high band in the cases (i) HB array only; (ii) $\mathrm{HB}$ array with unaltered LB element; (iii) $\mathrm{HB}$ array with choked LB element. It is clear that the unaltered LB element blocks HB electric field to a large extent, especially at low frequencies. The proposed choked LB element has much less effect on the field than the unaltered LB element. The resultant horizontal HB patterns after adding the choked LB element are shown in Fig. 15. Patterns for HB array alone are added for comparison. It is observed that the HB radiation patterns in the presence of choked LB element are almost the same as those of HB array alone, demonstrating the effectiveness of the chokes in reducing $\mathrm{HB}$ pattern distortion across the band. 


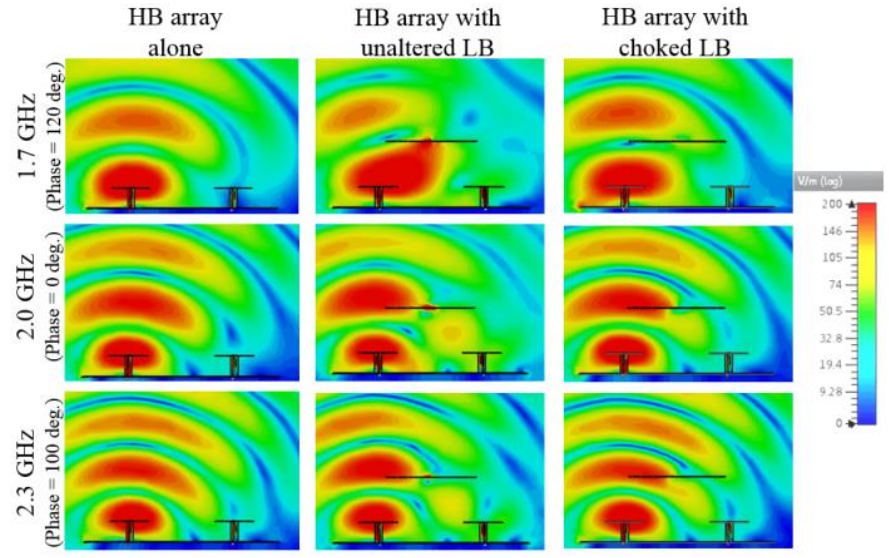

Fig. 14. The E-field cuts in the xoz-plane under the circumstances of i) only HB array, ii) $\mathrm{HB}$ array with unaltered LB radiators, and iii) $\mathrm{HB}$ array with choked $\mathrm{LB}$ radiators.

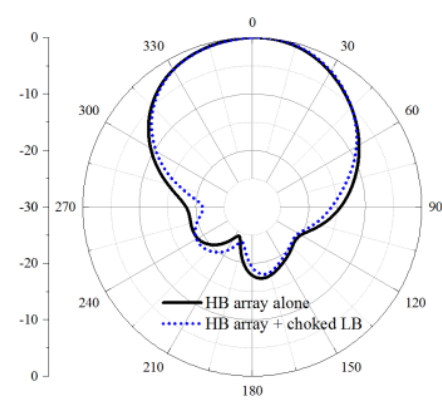

(a)

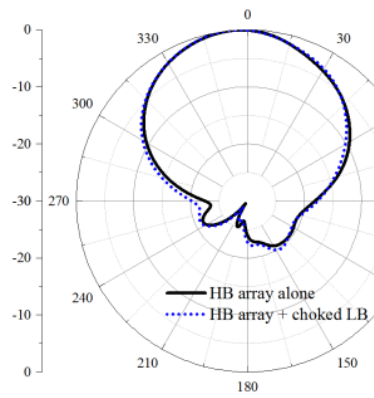

(c)

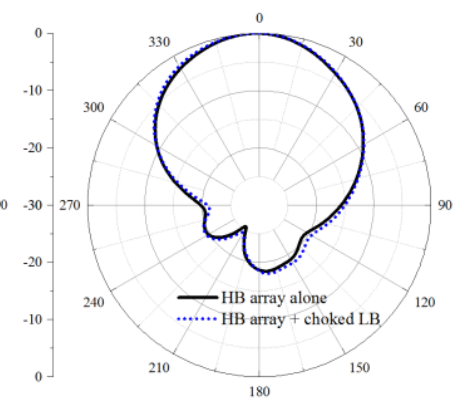

(b)
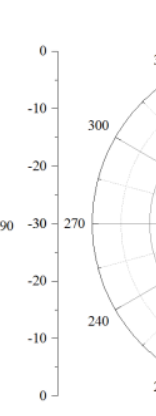

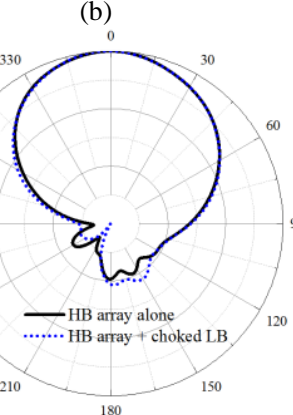

(d)
Fig. 15. Comparison of $\mathrm{HB}$ radiation patterns under the circumstances of i) only $\mathrm{HB}$ array, ii) HB array with choked LB radiators at (a) $1.7 \mathrm{GHz}$, (b) $1.9 \mathrm{GHz}$, (c) $2.1 \mathrm{GHz}$, and (d) $2.3 \mathrm{GHz}$.

TABLE I

OPTIMIZED PARAMETERS OF THE CHOKED ANTENNA

\begin{tabular}{cccc}
\hline \hline Parameters & Values (mm) & Parameters & Values (mm) \\
\hline$s 1$ & 16.75 & $s 2$ & 20.5 \\
$s 3$ & 22.25 & $s 4$ & 11 \\
$s 5$ & 9.5 & $s 6$ & 11 \\
$s 7$ & 7 & $g$ & 11.5 \\
$d$ & 3 & $w 1$ & 0.5 \\
$w 2$ & 4 & $w 3$ & 5.5 \\
\hline \hline
\end{tabular}

\section{Performance of the Choked LB Element}

As the realized chokes are not ideal and can only approximate a short circuit over a limited range of LB frequencies, they change the impedance properties of the LB element, making the impedance matching task more difficult. Nevertheless, following the guidelines of designing the impedance matching network given in [18], [19], a satisfactory matching result is obtained using baluns and impedance matching elements. Two specially designed baluns are orthogonally arranged to feed the two pairs of choked dipoles. The configuration of the choked LB element, together with the two baluns are shown in Fig. 16. The detailed parameters of the feed networks are listed in the Table II. Fig. 17 shows the matching results for the choked element. The antenna is matched to reflection coefficients $<-14 \mathrm{~dB}$ from $0.82 \mathrm{GHz}$ to $0.96 \mathrm{GHz}$. Compared with the unaltered LB element, the matching of the choked LB element is slightly degraded, but it is still satisfactory. The simulated radiation patterns of LB element with choked arms and unaltered arms at $0.82 \mathrm{GHz}, 0.88$ $\mathrm{GHz}, 0.92 \mathrm{GHz}$, and $0.96 \mathrm{GHz}$ are shown in Fig. 18. The patterns in the two cases are almost identical, showing that choking LB arms does not influence the radiation performance.

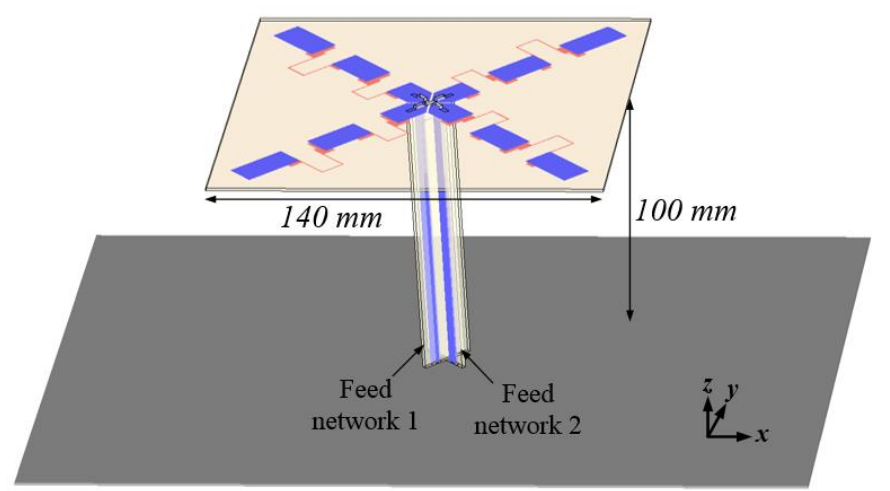

(a)

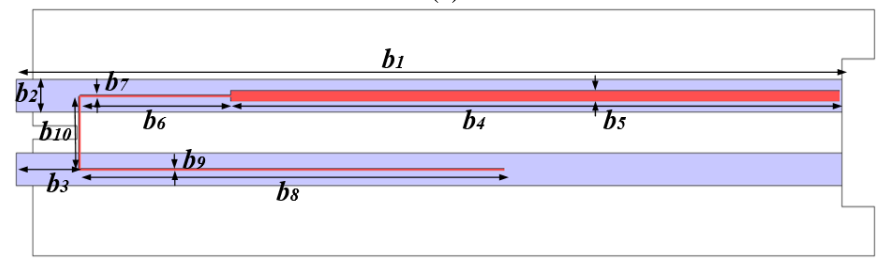

(a)

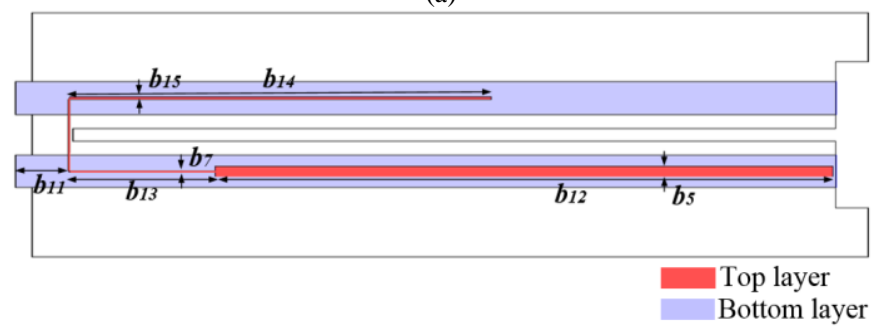

(b)

Fig. 16. (a) Perspective view of the choked LB antenna. Configuration of (b) feed network 1, and (c) feed network 2. (The baluns are printed on both sides of a substrate with a dielectric constant of 4.4 , a loss tangent of 0.0025 , and a thickness of $1.5 \mathrm{~mm}$.)

TABLE II

OPTIMIZED PARAMETERS OF BALUNS FOR THE CHOKED ANTENNA 


\begin{tabular}{cccc}
\hline \hline Parameters & Values $(\mathrm{mm})$ & Parameters & Values $(\mathrm{mm})$ \\
\hline$b 1$ & 101 & $b 2$ & 4 \\
$b 3$ & 7.6 & $b 4$ & 72.98 \\
$b 5$ & 1.4 & $b 6$ & 20 \\
$b 7$ & 0.2 & $b 8$ & 50 \\
$b 9$ & 0.2 & $b 10$ & 9 \\
$b 11$ & 6.475 & $b 12$ & 74.08 \\
$b 13$ & 20 & $b 14$ & 50 \\
$b 15$ & 0.25 & &
\end{tabular}

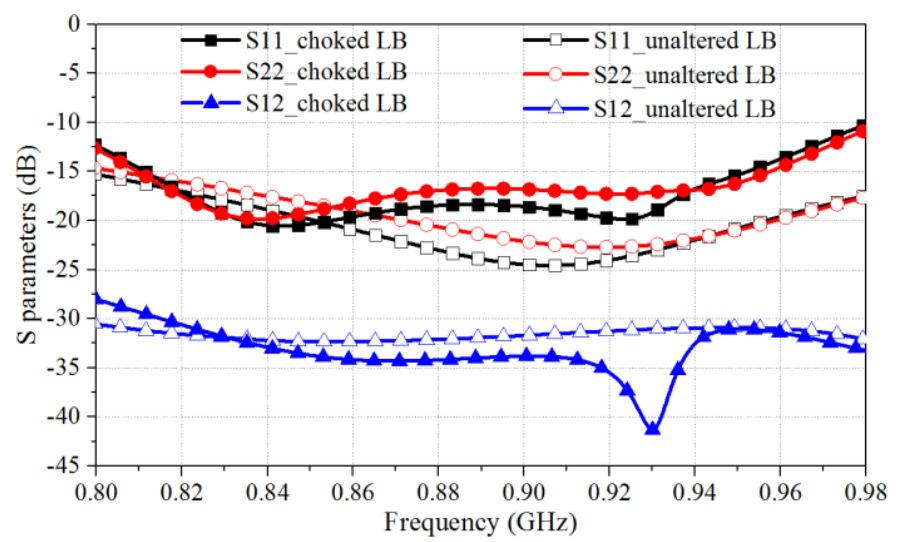

Fig. 17. S-parameters of the choked LB element and the unaltered LB element.

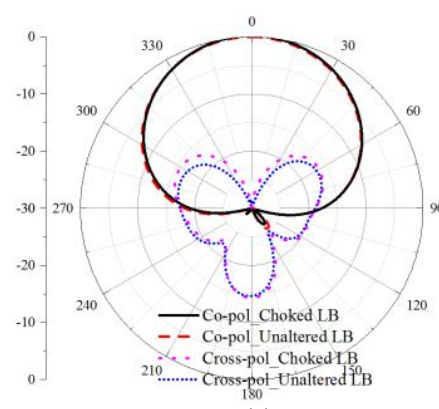

(a)

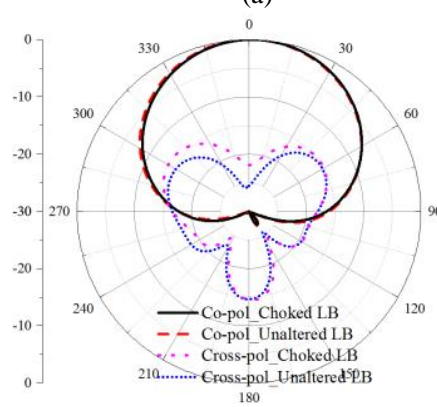

(c)

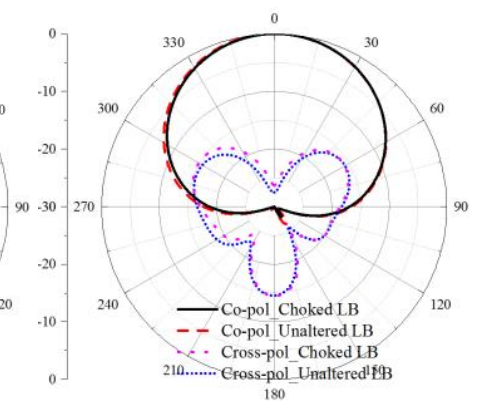

(b)
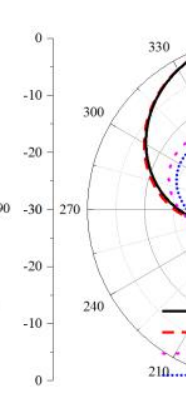

Fig. 18. Radiation patterns of the choked LB element and the unaltered LB element at (a) $0.82 \mathrm{GHz}$, (b) $0.88 \mathrm{GHz}$, (c) $0.92 \mathrm{GHz}$, and (d) $0.96 \mathrm{GHz}$.

\section{Simulated and Measured Results of the Dual- BAND DUAL-POLARIZED ARRAY USING THE CHOKED ELEMENT}

The prototype of the antenna array section was fabricated and tested, as shown in Fig. 19. Fig. 20 shows the simulated and measured reflection coefficients of the HB array. The results for the two HB arrays in the two columns are essentially the same due to the symmetric structure, so only the results for one column of the HB array are given. The HB return loss as expected is almost the same as obtained without the LB elements. Measured results agree well with the simulated ones, demonstrating good matching across a band of $28.6 \%$ from 1.71 $\mathrm{GHz}$ to $2.28 \mathrm{GHz}$. The radiation patterns for the $\pm 45^{\circ}$ polarization in one column at $1.7 \mathrm{GHz}, 2.0 \mathrm{GHz}$, and $2.3 \mathrm{GHz}$ are shown in Fig. 21. The simulated and measured patterns agree well. The simulated and measured cross polarization discrimination (XPD) at the boresight are $>20 \mathrm{~dB}$ and $>16 \mathrm{~dB}$, respectively. Fig. 22 shows the simulated and measured horizontal half-power beamwidth (HPBW) and realized gain of the HB array. For each polarization, the measured HPBW varies within $65^{\circ} \pm 5^{\circ}$, which shows that the array has very stable radiation performance as required in base station applications. The simulated and measured gain both vary from $10 \mathrm{dBi}$ to 12 dBi. The efficiencies of the HB ports are shown in Fig. 23. All the four ports have efficiencies higher than $90 \%$ across the operating band. The performance of HB array with choked LB is almost the same as that of HB array only.

The simulated and measured choked LB antenna results are shown in Figs. 24 to 27. As shown in Fig. 24, the measured bandwidth is $19.7 \%$ from $0.82 \mathrm{GHz}$ to $1.0 \mathrm{GHz}$ for reflection coefficients $<-10 \mathrm{~dB}$. The simulated and measured horizontal radiation patterns of the choked $\mathrm{LB}$ antenna at $0.82 \mathrm{GHz}, 0.88$ $\mathrm{GHz}$ and $0.96 \mathrm{GHz}$ are shown in Fig. 25. The simulated and measured radiation patterns agree well. The measured XPD is $>20 \mathrm{~dB}$ at boresight. The horizontal HPBW and gain are shown in Fig. 26. The simulated and measured HPBW varies within $71.5^{\circ} \pm 3.5^{\circ}$ and $69.5^{\circ} \pm 4^{\circ}$, respectively. The measured gain varies from $6.0 \mathrm{dBi}$ to $7.0 \mathrm{dBi}$, which is slightly less than the simulated gain. It is mainly caused by the loss of cables. The efficiencies of the LB antenna are shown in Fig. 27, which are higher than $89 \%$ across the band. Note that the presented gain results for HB and LB antennas are only for one array section. Higher gain can be obtained by repeating the array sections in the vertical direction ( $y$-direction). 


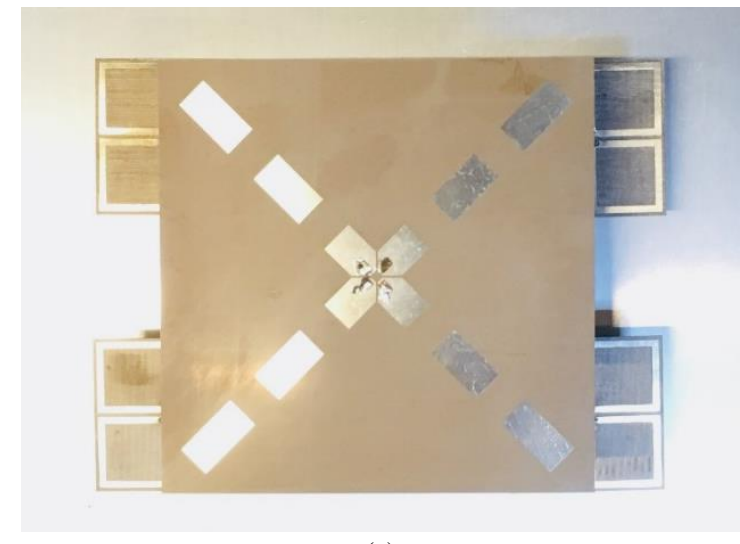

(a)

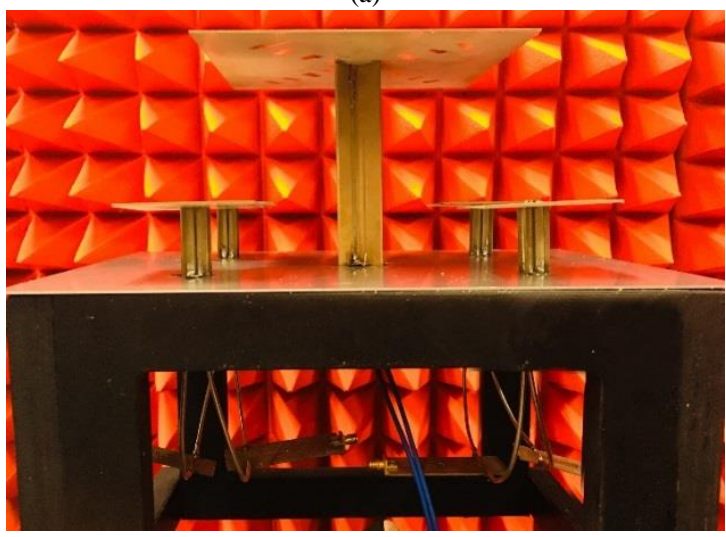

(b)

Fig. 19. (a) Top, and (b) side views of the dual-band dual-polarized interleaved array.

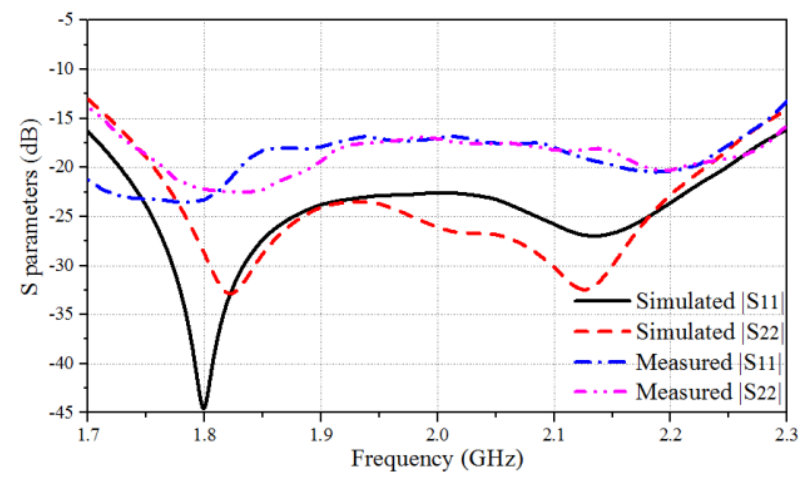

Fig. 20. Simulated and measured reflection coefficients of the HB array.

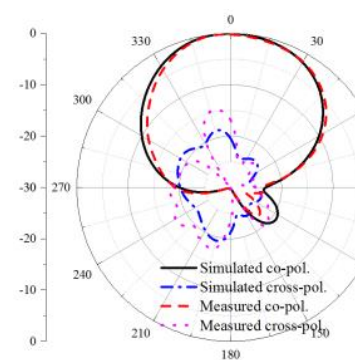

(a)

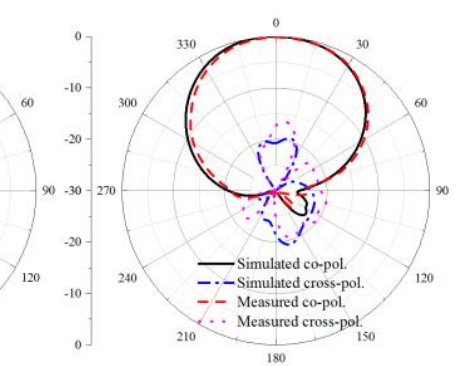

(b)

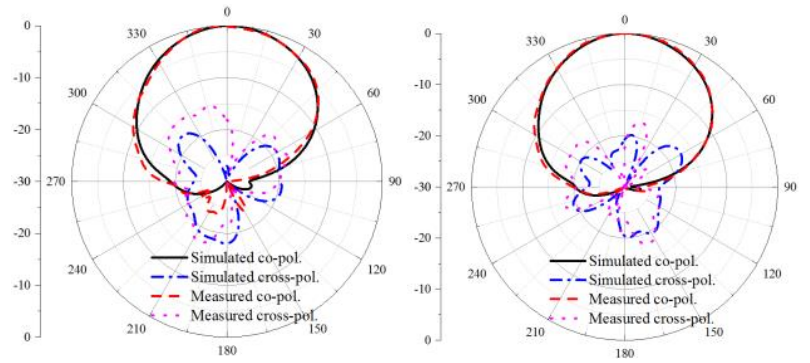

(c)

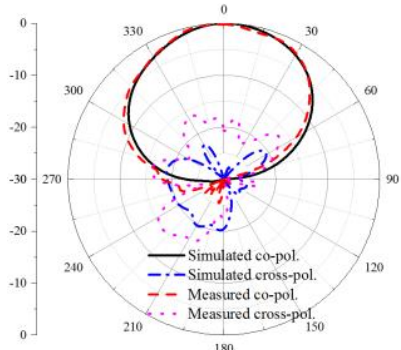

(e) (d)

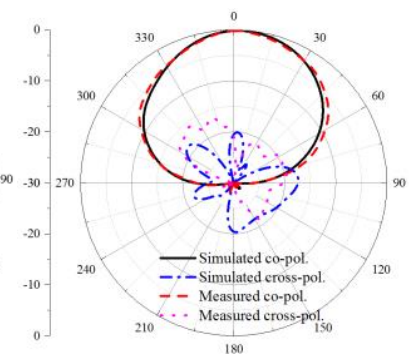

(f)

Fig. 21. Simulated and measured radiation patterns of the HB array. (a) Port 1 at 0.1.7 GHz. (a) Port 2 at $1.7 \mathrm{GHz}$. (c) Port 1 at $2.0 \mathrm{GHz}$. (d) Port 2 at $2.0 \mathrm{GHz}$. (e) Port 1 at $2.3 \mathrm{GHz}$. (f) Port 2 at $2.3 \mathrm{GHz}$.

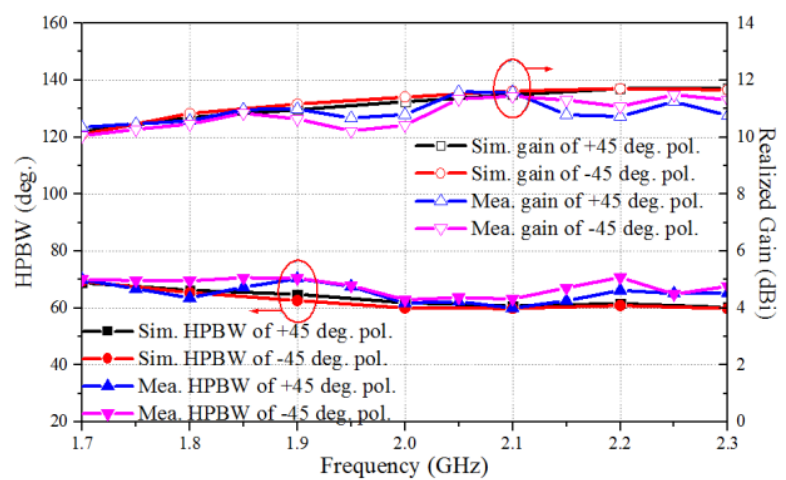

Fig. 22. Simulated and measured horizontal HPBW and gain of the HB array.

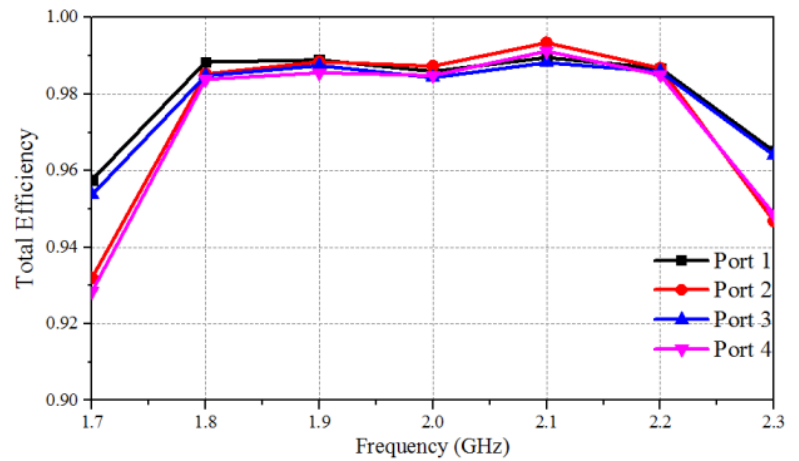

Fig. 23. Efficiencies for $\mathrm{HB}$ antenna ports $1-4$. 


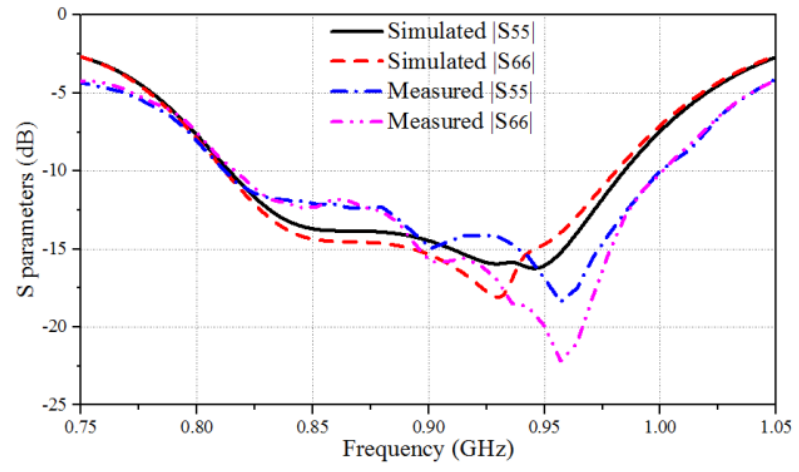

Fig. 24. Simulated and measured S-parameters of the choked LB antenna.

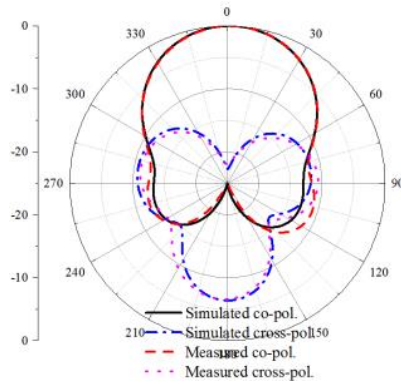

(a)

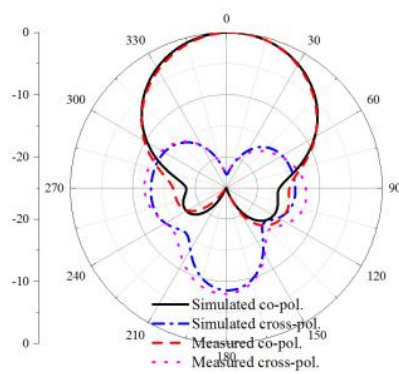

(c)

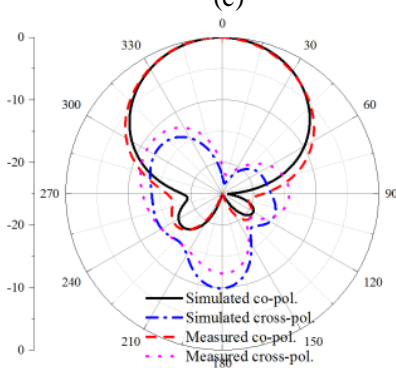

(e)

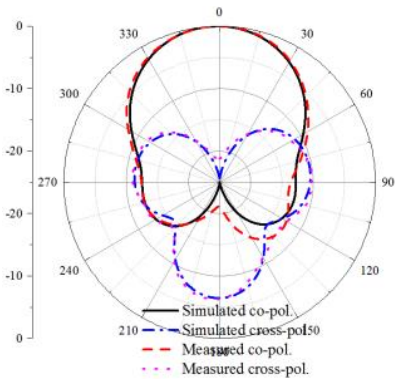

(b)

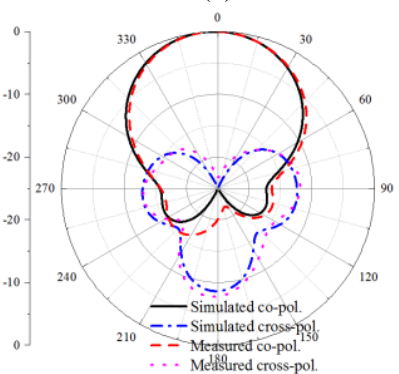

(d)

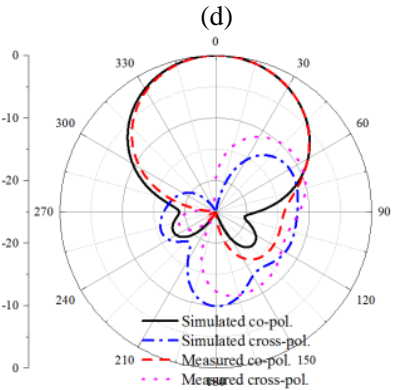

(f)
Fig. 25. Simulated and measured radiation pattern of the choked LB antenna. (a) Port 1 at $0.82 \mathrm{GHz}$. (b) Port 2 at $0.82 \mathrm{GHz}$. (c) Port 1 at $0.88 \mathrm{GHz}$. (d) Port 2 at $0.88 \mathrm{GHz}$. (e) Port 1 at $0.96 \mathrm{GHz}$. (f) Port 2 at $0.96 \mathrm{GHz}$.

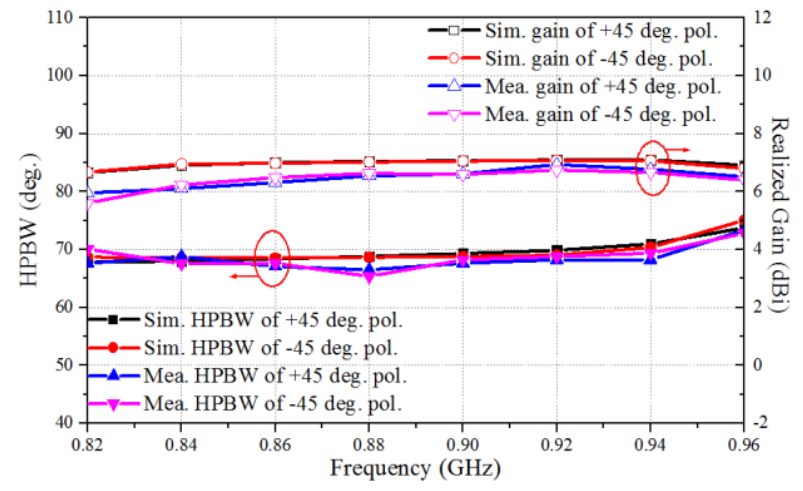

Fig. 26. Simulated and measured HPBW and gain of the choked LB antenna.

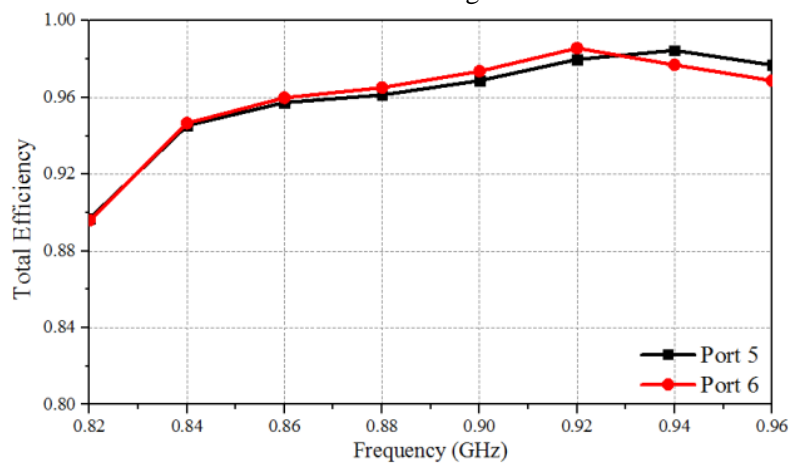

Fig. 27. Efficiencies for LB antenna ports 5 and 6.

\section{CONCLUSION}

In this paper, a method of suppressing cross-band scattering by choking the LB elements in an interleaved multiband antenna array is presented. The technique is demonstrated and analyzed in the context of a dual-band dual-polarized interleaved base station array configuration covering bands of $0.82 \mathrm{GHz}$ to $1.0 \mathrm{GHz}$ and $1.71 \mathrm{GHz}$ to $2.28 \mathrm{GHz}$. Simulated and measured results both demonstrate that choking the LB element largely restores the HB radiation pattern. The chokes have some effects on the LB impedance characteristics but satisfactory performance can still be obtained with suitable choice of choke impedances and optimized feed networks. The realized array section has stable radiation patterns in both the high and low bands with a compact size. Similar choking methods can be adopted to solve cross-band scattering issue in other multiband antenna systems.

\section{ACKNOWLEDGMENT}

The authors would like to thank Prof. Zheng Li at the Beijing Jiaotong University for his valuable suggestions on this work.

\section{REFERENCES}

[1] J. C. Soric, A. Monti, A. Toscano, F. Bilotti, and A. Alù, "Dual-polarized reduction of dipole antenna blockage using mantle cloaks, "IEEE Trans. Antennas Propag., vol. 63, no. 11, pp. 4827-4834, Nov. 2015.

[2] Y. He, Z. Pan, X. Cheng, Y. He, J. Qiao and M. M. Tentzeris, "A novel dual-band, dual-polarized, miniaturized and low-profile base station antenna," IEEE Trans. Antennas Propag., vol. 63, no. 12, pp. 5399-5408, Dec. 2015.

[3] H. Huang, Y. Liu and S. Gong, "A novel dual-broadband and dual-polarized antenna for $2 \mathrm{G} / 3 \mathrm{G} / \mathrm{LTE}$ base stations," IEEE Trans. Antennas Propag., vol. 64, no. 9, pp. 4113-4118, Sept. 2016.

[4] H. Huang, Y. Liu and S. Gong, "A dual-broadband, dual-polarized base station antenna for 2G/3G/4G applications," IEEE Antennas Wirel. Propag. Lett., vol. 16, pp. 1111-1114, 2017.

[5] G. Cui, S. -G. Zhou, G. Zhao, and S. -X. Gong, "A compact dual-band dualpolarized antenna for base station application" Prog. Electromagn. Res. C, vol. 64, pp. 61-70, 2016.

[6] Y. He, W. Tian and L. Zhang, "A novel dual-broadband dual-polarized electrical downtilt base station antenna for $2 \mathrm{G} / 3 \mathrm{G}$ applications," IEEE Access, vol. 5, pp. 15241-15249, 2017.

[7] Y. Liu, S. Wang, N. Li, J. Wang, and J. Zhao, "A compact dual-band dualpolarized antenna with filtering structures for sub- $6 \mathrm{GHz}$ base station applications", IEEE Antennas Wirel. Propag. Lett., vol. 17, iss. 10, pp. 1111-1114, Oct. 2018.

[8] Y. - B. Jung and S. -Y. Eom, "A compact multiband and dual-polarized mobile base-station antenna using optimal array structure", Int $J$ Antennas Propag., 2015. 
[9] L. Y. Nie, X. Q. Lin, Y. J. Chen, J. Zhang, B. Wang, Z. Q. Yang, and Y. Fan, "A low-profile coplanar dual-polarized and dual-band base station antenna array", IEEE Trans. Antennas Propag., early access, 2018.

[10] B. Jones, O. Isik, and C. Shang, "Dual-band interspersed cellular basestation antennas," EP. Patent 2769476 B1, Dec. 24, 2012.

[11] A. Monti, J. Soric, A. Alù, F. Bilotti, A. Toscano, and L. Vegni, "Overcoming mutual blockage between neighboring dipole antennas using a low-profile patterned metasurface," IEEE Antennas Wirel. Propag. Lett., vol. 11, pp. 1414-1417, 2012.

[12] Z. H. Jiang, P. E. Sieber, L. Kang, and D. H. Werner, "Restoring intrinsic properties of electromagnetic radiators using ultralightweight integrated metasurface cloaks," Adv. Funct. Mater., vol. 25, no. 29, pp. 4708-4716, Aug. 2015.

[13] A. Monti et al., "Mantle cloaking for co-site radio-frequency antennas," Appl. Phys. Lett., vol. 108, no. 11, p. 113502, 2016.

[14] H. M. Bernety and A. B. Yakovlev, "Reduction of mutual coupling between neighboring strip dipole antennas using confocal elliptical metasurface cloaks," IEEE Trans. Antennas Propag., vol. 63, no. 4, pp. 1554-1563, Apr. 2015.

[15] S. Vellucci, A. Monti, M. Barbuto. A. Toscano, and F. Bilotti, "Satellite applications of electromagnetic cloaking," IEEE Trans. Antennas Propag., vol. 65, no. 9, pp. 4931-4934, Jul. 2017.
[16] Y. Cui, R. Li, and H. Fu, "A broadband dual-polarized planar antenna for 2G/3G/LTE base stations," IEEE Trans. Antennas Propag., vol. 62, no. 9, pp. 4836-4840, Sept. 2014.

[17] C. Ding, H. Sun, R. W. Ziolkowski and Y. J. Guo, "Simplified tightlycoupled cross-dipole arrangement for base station applications," IEEE Access, vol. 5, pp. 27491-27503, Nov. 2017.

[18] H. Sun, C. Ding, B. Jones and Y. J. Guo, "A wideband base station antenna element with stable radiation pattern and reduced beam squint," IEEE Access, vol. 5, pp. 23022-23031, Oct. 2017.

[19] C. Ding, B. Jones, Y. J. Guo and P. Y. Qin, "Wideband matching of fullwavelength dipole with reflector for base station," IEEE Trans. Antennas Propag., vol. 65, no. 10, pp. 5571-5576, Oct. 2017. 